\title{
Model-based Estimation of Internal Heart Power in Aortic Valve Disease Patients
}

\author{
Matthias A.F. Gsell ${ }^{1}$, Gernot Plank ${ }^{1}$ \\ ${ }^{1}$ Institute of Biophysics, Medical University Graz, Graz, Austria
}

\begin{abstract}
Aortic valve disease (AVD) causes pressure overload of the left ventricle $(L V)$ which may trigger adverse remodeling and, eventually, precipitate progression towards heart failure $(H F)$. A frequent therapy is transvenous aortic valve implant (TAVI) which aims at reducing the transvalvular pressure gradient. However, TAVI does not always reverse HF symptoms. We aim to develop personalized computer models of LV electromechanics (EM), suitable for predicting acute TAVI-induced changes in $L V$ function and derive clinical markers such as internal heart power (IHP) which are believed to offer prognostic value of longer term outcomes. EM simulations of a heartbeat fitted to pre-treatment conditions were carried out and validated against complementary clinical data that were not used for model fitting. Based on the assumption of a reduced transvalvular pressure gradient $<20 \mathrm{mmHg}, a$ change in characteristic impedance of the aorta was estimated to simulate post-treatment conditions. IHP computed for pre- and post-treatment conditions was compared against the image-based clinical estimation of these quantities. All in silico models replicated clinical markers within prescribed margins of accuracy. Model predictions of IHP were comparable to the clinically derived analog.
\end{abstract}

\section{Introduction}

Elevated pressure gradients across the aortic valve as they occur in AVD impose a higher load upon the LV. Under such conditions the pressure produced by the LV must increase in order to produce an adequate cardiac output that meets the metabolic demands. This requires higher active forces generated by the LV wall, which can be achieved either by an increase in wall stresses or ventricular mass. Such pressure overload conditions, if persistent for long enough, trigger adverse remodeling and, eventually, precipitate progression towards HF. Treatments for alleviating pressure overload conditions aim at reducing the elevated pressure gradients closer to normal levels. Transvalvular pressure gradients in AVD patients can be treated by interventions such as TAVI. However, even though these interventions accomplish a significant reduction of the pressure gradient, depending on the specific etiology, they do not necessarily lead to a reversal of HF symptoms. Thus a successful reduction of a pathologically elevated pressure gradients alone cannot be a reliable prognostic marker of long-term post-treatment morbidity and mortality in patients suffering from LV pressure overload and its consequences. In general, currently used clinical biomarkers are limited in their predictive power with regard to post-treatment outcomes of interventions altering afterload such as TAVI. Consequently, alternative biomarkers are sought after which provide a complementary view on cardiac function and, offer a higher predictive power with regard to longer term outcomes. Wall stresses are directly linked to the internal power generated by the cardiac muscle and the work performed by it and as such can be considered a metabolic marker. Despite the diagnostic potentials of markers based on wall stresses and IHP, their assessment has not evolved towards a routinely used diagnostic tool in the clinic yet mainly due to methodological limitations. In this study patient-specific high resolution biomechanical FE models are used to compute the IHP for the pre- and post-treatment situation and the in silico results are compared to their clinical analog.

\section{Methods}

\subsection{Building anatomical $L V$ models}

The LV model generation is based on a variety of datasets. For the mesh generation we use 3D whole heart MRI scans acquired at end diastole (ED) and the multilabel segmentation of these scans. Dataset were acquired at the German Heart Center Berlin (DHZB). Due to the low resolution of the segmentation, an up-sampling and smoothing algorithm is applied to obtain a high resolution segmentation. The aortic wall is automatically added, see Figure 1. The high resolution segmentation is meshed using the CGAL library (https://www.cgal.org). To generate the fibers, a rule-based method [1] is applied, see Figure 2. 

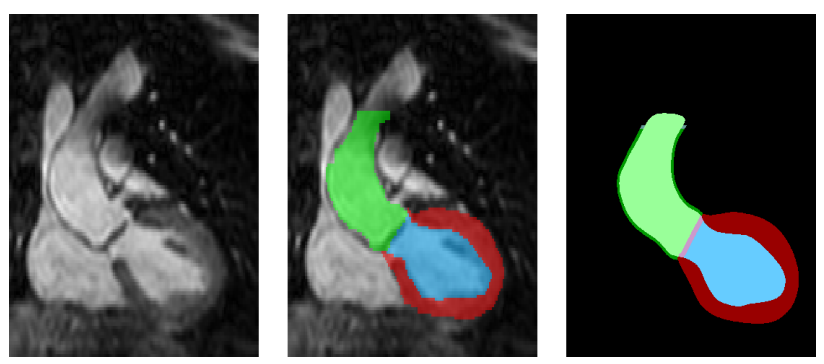

Figure 1. MRI scan, segmentation and smoothing.
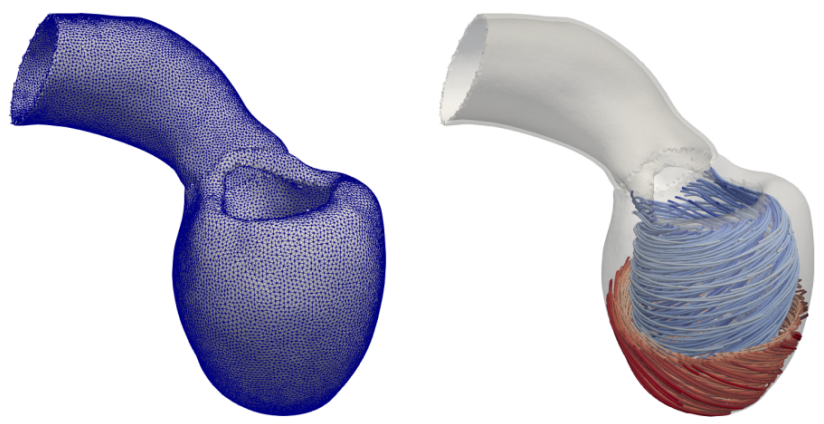

Figure 2. Mesh generation and fiber orientation.

\subsection{Modeling mechanical deformation}

The mechanical deformation is simulated by solving a quasi static boundary value problem (BVP), which is a simplification of Cauchy's equation of motion, see [2]. For a given pressure $p(t)$, find the unknown displacement $\mathbf{u}$, such that

$$
\begin{aligned}
-\nabla \cdot \sigma_{\text {tot }}(\mathbf{u}, t) & =0 & & \text { in } \Omega \\
\sigma_{\text {tot }}(\mathbf{u}, t) \mathbf{n} & =-p(t) \mathbf{n} & & \text { on } \Gamma_{N} \\
\sigma_{\text {tot }}(\mathbf{u}, t) \mathbf{n} & =0 & & \text { on } \Gamma_{H} \\
\mathbf{u} & =0 & & \text { on } \Gamma_{D}
\end{aligned}
$$

holds for $t \in[0, T]$. By $\Omega$ we denote the deformed geometry and by $\Gamma:=\partial \Omega$ its boundary with $\Gamma=\overline{\Gamma_{D}} \cup \overline{\Gamma_{H}} \cup \overline{\Gamma_{N}}$ and $\left|\Gamma_{D}\right|>0$. The normal outward vector of $\Gamma$ is denoted by $\mathbf{n}$. The total stress in our model is the sum of a given active stress and a passive stress, i.e. $\sigma_{\text {tot }}=\sigma_{\text {act }}+\sigma_{\text {pas }}$ with

$$
\begin{aligned}
& \sigma_{\text {act }}=J^{-1} \mathbf{F}\left(S_{a}\left(\mathbf{f}_{0}^{\top} \mathbf{C f}_{0}\right)^{-1} \mathbf{f}_{0} \otimes \mathbf{f}_{0}\right) \mathbf{F}^{\top} \\
& \sigma_{\text {pas }}=J^{-1} \mathbf{F}\left(2 \frac{\partial \Psi(\mathbf{C})}{\partial \mathbf{C}}\right) \mathbf{F}^{\top}
\end{aligned}
$$

where $\mathbf{F}$ is the deformation gradient, $J$ its Jacobian and the right Cauchy-Green tensor C. $S_{a}$ is the scalar contractile stress generated by the myocytes acting along fiber direction $\mathbf{f}_{0}$ in Lagrange description. To determine $S_{a}$, an efficient EP model is used to generate the activation sequences which serve as a trigger for the active stress, see [3]. The LV myocardium was characterized as a hyperelastic, nearly incompressible transversally orthotropic material with a nonlinear stress-strain relationship $\Psi$. The incompressibility is realized by splitting the strain energy function $\Psi$ into a isochoric part and a volumetric part, i.e. $\Psi=\Psi_{\text {iso }}+\Psi_{\text {vol }}$ with $\Psi_{\text {vol }}:=\frac{\kappa}{2}(\log (J))^{2}$. To compute the approximate solution of the BVP (1), we use our in-house software CARP [4] which is based on the finite element method (FEM).

\subsection{Fitting the mechanics model}

\subsubsection{Unloading}

As described in Section 2.1, the anatomical model is based on MRI scans acquired at ED. At that moment a physiological pressure load is present and thus, the model we generated is not stress free. For the stress free configuration with material coordinates $\mathrm{x}^{\star}$ and in vivo stress $\sigma^{\star}$, there holds $\Omega\left(\mathbf{x}_{m}, \sigma^{\star}\right)=\mathcal{S}\left(\Omega\left(\mathbf{x}^{\star}, \mathbf{0}\right), p_{m}\right)$ where $\mathcal{S}$ is an appropriate forward mechanics solver, $\mathbf{x}_{m}$ are the measured material coordinates and $p_{m}$ is the measured pressure at ED. This deformation defines a mapping $\Phi$ : $\mathbf{x}^{\star} \mapsto \mathbf{x}_{m}$, see Figure 3. Applying the inverse mapping

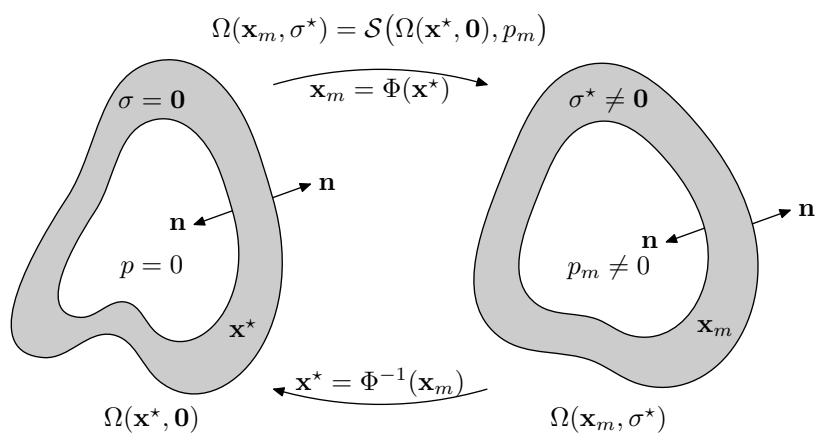

Figure 3. Unloaded and loaded configuration.

$\Phi^{-1}$ to $\mathbf{x}_{m}$ yields $\mathbf{x}^{\star}$, which entails the representation $\Omega\left(\mathbf{x}_{m}, \sigma^{\star}\right)=\mathcal{S}\left(\Omega\left(\Phi^{-1}\left(\mathbf{x}_{m}\right), \mathbf{0}\right), p_{m}\right)$. An iterative approximation of the inverse mapping $\Phi^{-1}$ using a backward displacement method, see [5], provides the desired stress free configuration $\Omega\left(\mathbf{x}^{\star}, \mathbf{0}\right)$ and the in vivo stress $\sigma^{\star}$, see left hand side of Figure 4.

\subsubsection{Constitutive fitting}

To find the passive mechanical material properties, the end diastolic pressure-volume relation (EDPVR) is fitted to the empiric Klotz EDPVR, which can be estimated from a single measured pressure-volume (PV) pair, see [6]. The isochoric strain-energy function proposed by Guccione, see [7], is defined as $\Psi_{\text {iso }}=\frac{\alpha}{2}(\exp (Q)-1)$ with

$Q=b_{f} E_{f f}^{2}+2 b_{f t}\left(E_{f s}^{2}+E_{f n}^{2}\right)+b_{t}\left(E_{s s}^{2}+E_{n n}^{2}+2 E_{s n}^{2}\right)$ 
where $\alpha$ is a scaling factor and $b_{f}, b_{f t}, b_{t}$ are material parameters along the fibers (f), across the transverse planes $(\mathrm{t})$ and the fiber-transverse (ft) shear planes. To reduce the number of parameters to fit, we assign to $b_{f}, b_{f t}$ and $b_{t}$ the default values proposed in [7]. Thus, we only fit the parameter $\alpha$ by running a passive-inflation mechanics simulation and fitting the EDPVR to the Klotz EDPVR.

\subsubsection{Windkessel fitting}

The LV is coupled to a three-element Windkessel model, see [8], which simulates a lumped cardiovascular system (see right hand side of Figure 4). The Windkessel model is given by the equation

$$
\frac{\mathrm{dq}}{\mathrm{dt}}=\frac{p}{Z R C}+\frac{1}{Z} \frac{\mathrm{dp}}{\mathrm{dt}}-\left(\frac{1}{Z C}+\frac{1}{R C}\right) q
$$

with the flux $q:=-\frac{\mathrm{dV}_{\text {cav }}}{\mathrm{dt}}$ and the pressure $p$ in the LV. Since the serial resistance $Z$, the parallel resistance $R$ and the capacitance $C$ are not known a-priori, they have to be fitted. For this, we use personalized clinical datasets like volume traces and LV peak pressures. To obtain the Wind-
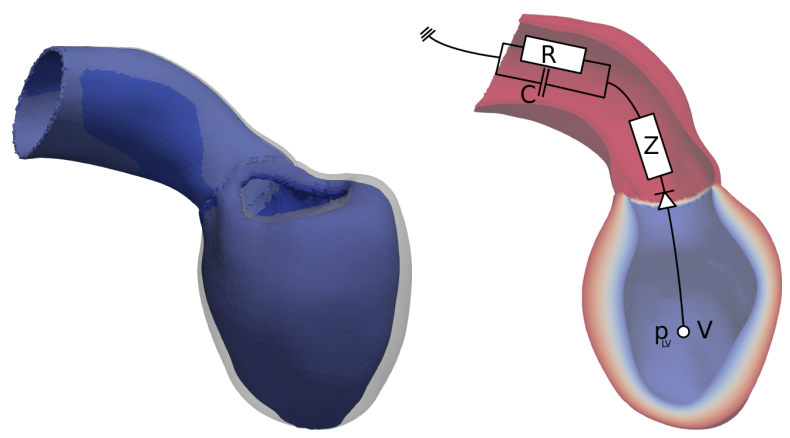

Figure 4. Unloaded (solid) and loaded (transparent) configuration, Windkessel coupling.

kessel parameters $Z, R$ and $C$, an optimization problem is derived and solved.

\subsection{Data analysis}

\subsubsection{Internal power and work}

Let $\mathbf{u}$ be the displacement at a time $t \in[0, T]$, then the internal heart power at $t$ can be expressed by

$$
P_{\text {int }}(t)=\int_{\Omega} \sigma_{\text {tot }}(\mathbf{u}, t): \dot{\varepsilon}(\mathbf{u}, t) \mathrm{dV}
$$

where $\dot{\varepsilon}$ denotes the strain rate tensor and $\sigma_{\text {tot }}$ as in Section 2.2, see [9]. Integrating the IHP yields the internal work

$$
W_{\text {int }}=\int_{0}^{T} P_{\text {int }}(t) \mathrm{dt} .
$$

\subsubsection{External work}

External work, or pressure-volume work, occurs when the volume of a closed system changes. The external work is given by the expression

$$
W_{\text {ext }}:=\int_{V_{\text {cav } 0}}^{V_{\text {cav } 1}} p\left(V_{\text {cav }}\right) \mathrm{d} V_{\text {cav }}
$$

where $p$ denotes the pressure and $V_{\text {cav }}$ denotes the enclosed volume.

\subsection{Adaptation to post-treatment case}

For the pre-treatment case, an EM simulation is done with parameters fitted as described in Section 2.3. For the post-treatment situation, we assume that the parallel resistance $R$ and the capacitance $C$, are the same as in the pretreatment case. Furthermore we suppose, that the serial resistance $Z$, which corresponds to the aortic valve, is less than the serial resistance in the pre-treatment case. Thus, we iteratively perform a parameter fit for a modified $Z$ followed by an EM simulation until we obtain the same stroke volume as in the pre-treatment situation.

\section{Results}

We applied the methods of Section 2 to four patients suffering from AVD. In Table 1 some patient characteristics are listed. Comparing simulated quantities to their clinic

\begin{tabular}{|c|cccc|}
\hline & Sex & Age[y] & EDV[ml] & ESV[ml] \\
\hline B0553-90 & F & 63 & 112.0 & 46.0 \\
B0343-91 & M & 73 & 121.0 & 54.7 \\
B0162-92 & M & 54 & 119.2 & 42.0 \\
B0358-93 & M & 85 & 172.0 & 103.0 \\
\hline
\end{tabular}

Table 1. Pretreatment patient characteristics.

analog allows a validation of the models. In Figure 6 the

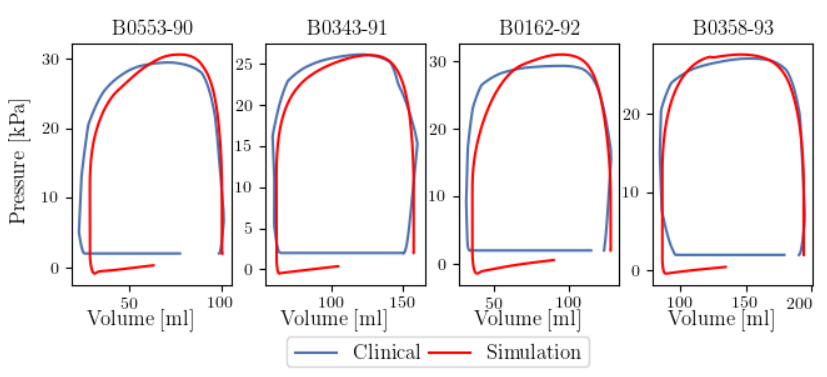

Figure 5. PV loops.

clinical PV loops are plotted against the PV loops from the simulation. In Figure 5 we plotted the end diastolic volume (EDV) and end systolic volume (ESV) for the pre- 


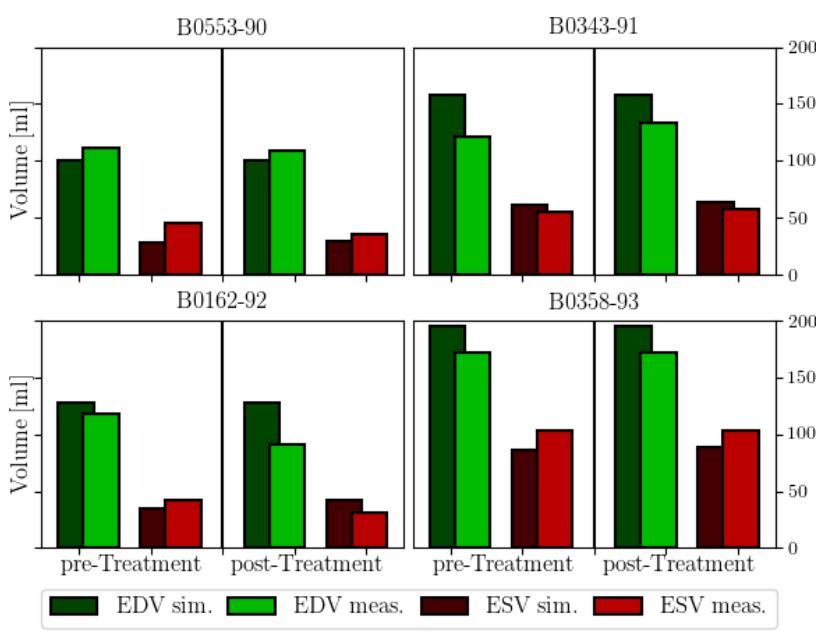

Figure 6. EDV and ESV.

and post-treatment cases. Since the pressure and volume were used for model fitting, they can only be used for assessing the quality of the fit. Figure 7 shows the internal work for the pre- and post-treatment cases.

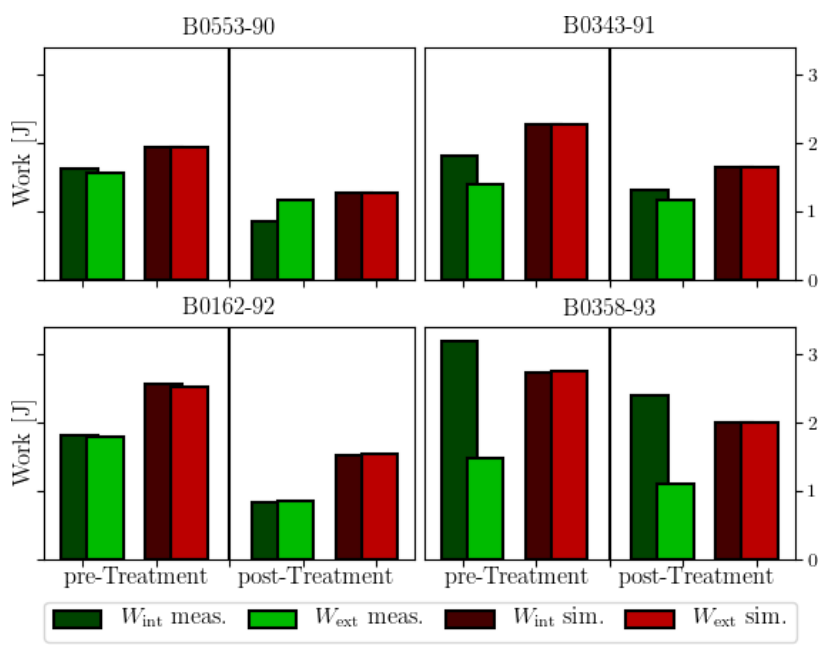

Figure 7. Internal and external work.

\section{Conclusion}

The presented methodology allows an efficient and highly automated execution of patient-specific EM models of the LV. The described workflow allows to build and run EM models for the pre- and post-treatment situations within 1-2 days. Since the work was not used for fitting, it can be used for an independent validation of model predictions. In Figure 7 prediction of a reduced IHP in the post-treatment situation relative to the pre-treatment situation is clearly recognizable.

\section{Acknowledgements}

This work was supported by the grant F3210-N19 from the Austrian Science Fund (FWF) within the SFB Mathematical Optimization and Applications in Biomedical Sciences and is part of the EU project CARDIOPROOF (partially funded by the European Commission under ICT2013.5.2, Grant Agreement: 611232)

\section{References}

[1] Bayer JD, Blake RC, Plank G, Trayanova NA. A novel rulebased algorithm for assigning myocardial fiber orientation to computational heart models. Annals of Biomedical Engineering Oct 2012;40(10):2243-2254. ISSN 1573-9686.

[2] Augustin CM, Neic A, Liebmann M, Prassl AJ, Niederer SA, Haase G, Plank G. Anatomically accurate high resolution modeling of human whole heart electromechanics: a strongly scalable algebraic multigrid solver method for nonlinear deformation. Journal of computational physics 2016; 305:622-646.

[3] Neic A, Campos FO, Prassl AJ, Niederer SA, Bishop MJ, Vigmond EJ, Plank G. Efficient computation of electrograms and ecgs in human whole heart simulations using a reactioneikonal model. Journal of Computational Physics 2017;

[4] Vigmond E, Dos Santos RW, Prassl A, Deo M, Plank G. Solvers for the cardiac bidomain equations. Progress in biophysics and molecular biology 2008;96(1):3-18.

[5] Bols J, Degroote J, Trachet B, Verhegghe B, Segers P, Vierendeels J. A computational method to assess the in vivo stresses and unloaded configuration of patient-specific blood vessels. Journal of Computational and Applied Mathematics 2013;246:10-17.

[6] Klotz S, Dickstein ML, Burkhoff D. A computational method of prediction of the end-diastolic pressure-volume relationship by single beat. Nature protocols 2007;2(9):2152.

[7] Guccione JM, McCulloch AD, Waldman L, et al. Passive material properties of intact ventricular myocardium determined from a cylindrical model. J Biomech Eng 1991; 113(1):42-55.

[8] Westerhof N, Elzinga G, Sipkema P. An artificial arterial system for pumping hearts. Journal of applied physiology 1971;31(5):776-781.

[9] Holzapfel GA. Nonlinear solid mechanics. John Wiley \& Sons, Ltd., Chichester, 2000. ISBN 0-471-82304-X. A continuum approach for engineering.

Address for correspondence:

Dr. Matthias A.F. Gsell

Institut für Biophysik

Neue Stiftingtalstraße 6/IV

8010 Graz, Austria

matthias.gsell@medunigraz.at 